\title{
THE MASS-SFR-METALLICITY RELATION OF STAR FORMING GALAXIES AND ITS EVOLUTION: IMPLICATIONS FOR GRB/SN HOST GALAXIES
}

\author{
Y. Niino ${ }^{1}$
}

\begin{abstract}
Observed properties of gamma-ray burst (GRB) host galaxies are important clues to understand the nature of GRB progenitors. However, the properties of the host galaxies don't directly tell us the nature of the GRB progenitors, and the decipherment of the clue relies on our understanding of general galaxies. The relation between stellar mass, star formation rate (SFR), and metallicity of star forming galaxies (so called the fundamental metallicity relation) has recently attracted attention regarding its possible impact upon our understanding on the properties of the long GRB host galaxies. In this study, I show the possibility of redshift evolution of the mass-SFR-metallicity relation which has been claimed to be independent of redshift, and discuss implications of the evolving relation for the properties of GRB/SN host galaxies.
\end{abstract}

\section{Introduction}

The stellar mass-metallicity $\left(M_{\star}-Z\right)$ relation of the long gamma-ray burst (GRB) host galaxies is shifted toward low-metallicity compared to that of the field star forming galaxies (Levesque et al. 2010). High SFR galaxies have lower $Z$ than low SFR galaxies with similar mass (Mannucci et al. 2010), and hence the shift of the $M_{\star}-Z$ relation of the long GRB host galaxies may be a result of the SFR $-Z$ correlation (Kocevski \& West 2011; Mannucci et al. 2011). Galaxies at wide range of redshifts $(0.1 \lesssim z \lesssim 3)$ agree with a single $M_{\star}-\mathrm{SFR}-Z$ relation, and hence the relation is claimed to be independent of redshift.

If the shift of the $M_{\star}-Z$ relation of the long GRB host galaxies is a result of the SFR $-Z$ correlation, host galaxies of other SFR tracing transients are expected to have similar shift of the $M_{\star}-Z$ relation. However the $M_{\star}-Z$ relation of Type II supernova (SN) host galaxies is not shifted (Stoll et al. 2012).

${ }^{1}$ Division of Optical and Near-Infrared Astronomy, National Astronomical Observatory of Japan, 2-21-1 Osawa, Mitaka, Tokyo, Japan 

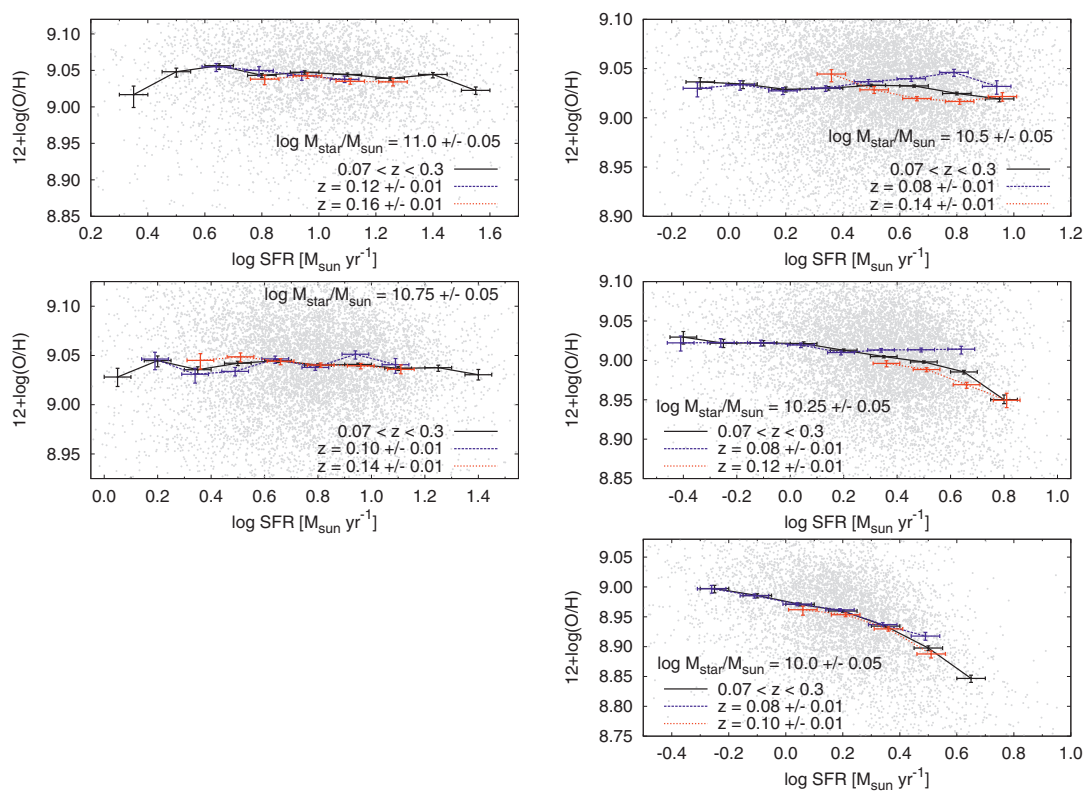

Fig. 1. The SFR $-Z$ relations at various $M_{\star}$. The systematic redshift dependence is found in the mass ranges $M_{\star}<10^{10.5} M_{\odot}$.

\section{Evolution of the $M_{\star}-S F R-Z$ Relation}

The $M_{\star}-\mathrm{SFR}-Z$ relation is originally defined by galaxies in the Sloan Digital Sky Survey (SDSS) spectroscopic sample at $z \sim 0.1$. Within the redshift range of the SDSS galaxies $(0.07 \leq z \leq 0.3)$, galaxies with larger $M_{\star}$ and SFR reside at higher redshifts. The galaxies at $z>0.3$ which are confirmed to be consistent with the relation also have higher SFR at higher redshifts. Hence in the previous studies of the $M_{\star}-\mathrm{SFR}-Z$ relation, SFR and redshift of galaxies are degenerate. Thus it is not clear whether the metallicity is dependent on SFR or redshift.

If the $M_{\star}-\mathrm{SFR}-Z$ relation actually evolves with redshift, the evolution may explain the difference between the $M_{\star}-Z$ relations of the long GRB host galaxies and the type II SN host galaxies. Type II SNe are typically at lower redshifts than the low redshift sample of long GRBs. Separating the SDSS galaxies into narrow redshift bins, we find difference of the $M_{\star}-\mathrm{SFR}-Z$ relation between different redshifts in the mass ranges $M_{\star}<10^{10.5} M_{\odot}$ (Fig. 1, see Niino 2012 for detail).

\section{Testing artificial effects}

In this section, we test artificial effects that may produce seeming evolution of the $M_{\star}-\mathrm{SFR}-Z$ relation using the SDSS galaxies with $M_{\star}=10^{10.5 \pm 0.05} M_{\odot}$ and $\mathrm{SFR}=10^{0.8 \pm 0.05}\left[M_{\odot} / \mathrm{yr}\right]$ (see the top-right panel of Fig. 1 ). 


\subsection{The fiber aperture effect}

The spectroscopic fiber covers lager area of a galaxy at higher redshifts, and metallicity of a galaxy is often lower in the outskirts than at the center. Thus the difference of fiber covering fraction may cause seeming difference of metallicity.

We investigate the correlation between the fiber covering fraction and the metallicity of galaxies. Unlike expected from the metallicity gradient, galaxies with larger fiber covering fraction have higher metallicity. We note that galaxies with larger radius have smaller metallicity (Ellison et al. 2008). The positive correlation between the fiber covering fraction and the metallicity suggests that the fiber covering fraction is determined by the intrinsic radius of galaxies rather than their redshift. We also note that it is difficult to explain why we find the evolution only in the low mass range, by the fiber aperture effect.

\subsection{Sampling bias due to the limiting magnitude}

The galaxies in the SDSS sample are brighter at higher redshifts, due to the limiting magnitude of the spectroscopic target selection. The difference of metallicity may results from the difference of luminosity.

We separate the low redshift galaxies $(z \sim 0.8)$ into bright and faint subsamples (at $\left.M_{r}=-21.5\right)$. The bright and faint subsamples have consistent metallicity distributions to each other, while the metallicity distributions of the bright subsample and the high redshift $(z \sim 1.4)$ sample are inconsistent to each other. Note that the low redshift bright sample galaxies have similar $M_{r}$ to those of the high redshift sample galaxies.

\section{Conclusions}

The $M_{\star}-\mathrm{SFR}-Z$ relation is different between $z<0.1$ and $>0.1$. This redshift evolution is hardly explained by the observational effects. This evolution of the $M_{\star}-\mathrm{SFR}-Z$ relation could explain the difference of the $M_{\star}-Z$ relations between the host galaxies of the Type II SNe and long GRBs.

\section{References}

Ellison, S.L., Patton, D.R., Simard, L., \& McConnachie, A.W., 2008, ApJ, 672, L107

Kocevski, D., \& West, A.A., 2011, ApJ, 735, L8

Levesque, E.M., Kewley, L.J., Berger, E., \& Zahid, H.J., 2010, AJ, 140, 1557

Mannucci, F., Cresci, G., Maiolino, R., et al., 2010, MNRAS, 408, 2115

Mannucci, F., Salvaterra, R., \& Campisi, M.A., 2011, MNRAS, 414, 1263

Niino, Y., 2012, ApJ, 761, 126

Stoll, R., Prieto, J.L., Stanek, K.Z., \& Pogge, R.W., 2012 [arXiv:1205. 2338] 
